\title{
Recurrent Hypocalcemic Tetany as the Initial Presentation in Some Persons with Hyperthyroidism: A Case Series
}

\author{
Yakubu Lawal $^{\mathrm{a}} \quad$ Umar Faruk Dahuwa $^{\mathrm{a}} \quad$ Ibrahim Abdullahi $^{\mathrm{a}}$ \\ Felicia Ehusani Anumah ${ }^{\mathrm{b}}$ \\ aDepartment of Internal Medicine, Federal Medical Center Azare, Azare, Nigeria; \\ ${ }^{b}$ Department of Internal Medicine, College of Health Sciences, University of Abuja, Abuja, Nigeria
}

\section{Keywords}

Hyperthyroidism $\cdot$ Hypocalcemia $\cdot$ Tetany

\begin{abstract}
This is a case series of recurrent hypocalcemic tetany as the initial presentation in some cases of hyperthyroidism. Literature review has revealed more reports of hypercalcemia than hypocalcemia as a feature of hyperthyroidism. The recurrence of hypocalcemic tetany in the 3 cases reported ceased following the treatment of hyperthyroidism. The aim of this case series is to alert clinicians to the fact that hypocalcemic tetany can be the initial clinical presentation of hyperthyroidism. It also highlights the resilience of managing endocrine patients in resource-poor settings with the aid of high clinical acumen and fewer-than-expected laboratory investigations. Further research is needed to investigate our hypothesis that disordered calcium metabolism in thyrotoxicosis may manifest in successive phases of hypercalcemia-normocalcemiahypocalcemia. The rate at which individual thyrotoxic patient traverses these phases may be directly proportional to the background vitamin $D$ deficiency, negative calcium balance, and bone mineral density.

(c) 2021 The Author(s)

Published by S. Karger AG, Basel
\end{abstract}

\section{Introduction}

Hyperthyroidism is known to cause a relatively mild hypercalcemia secondary to an increase in bone turnover and mobilization of calcium from bone into the circulation [1]. This is mediated predominantly by thyroid hormone receptor- $\alpha$ [2]. Previous reports from the West also showed that hyperthyroid persons have normal or increased serum total calcium [3-5].

However, a few studies especially from India revealed that hypocalcemia is a feature of hyperthyroidism in some persons $[6,7]$. This was attributed to background vitamin $\mathrm{D}$ deficiency among the Indians studied [6-9]. Dhanwal [9] postulated that increased skin pigmentation and related vitamin D deficiency among some Indians may contribute to abnormal bone mineral homeostasis in thyrotoxic persons. Nonetheless, many investigators have reported normal serum levels of $25(\mathrm{OH}) \mathrm{D}$ in hyperthyroidism $[10,11]$.

The aim of this case series is to enlighten clinicians that hypocalcemic tetany can be the initial presentation of hyperthyroidism. Thus, a high index of suspicion to pursue the diagnosis of thyrotoxicosis must be developed in cases of recurrent hypocalcemia. This case series also seek to karger@karger.com www.karger.com/dde

Karger $\stackrel{\text { ' }}{=}$ BOPEN ACCESS
(C) 2021 The Author(s)

Published by S. Karger AG, Basel

This is an Open Access article licensed under the Creative Commons Attribution-NonCommercial-4.0 International License (CC BY-NC) (http://www.karger.com/Services/OpenAccessLicense), applicable to the online version of the article only. Usage and distribution for commercial purposes requires written permission.
Correspondence to:

Yakubu Lawal, lawalyaqub2006@yahoo.com 
highlight the resilience of clinical endocrinologists in managing endocrine patients in resource-poor settings with the aid of high clinical acumen and fewer-than-expected laboratory investigations.

Literature search was done using Google Scholar, PubMed, and PubMed Central. Search terminologies used include "hypocalcemia in thyrotoxicosis or hyperthyroidism," "calcium disorders in thyrotoxicosis or hyperthyroidism," and "disordered calcium metabolism in thyrotoxicosis or hyperthyroidism."

\section{Case Report 1}

JA is a 30-year-old woman who presented with a recurrent history of carpopedal spasm over the previous 2 years. On each occasion, she was managed with initial intravenous calcium, then maintained on calcium supplements.

During the last episode of carpopedal spasm, detailed history revealed the patient had heat intolerance and palpitations, though no significant weight loss, and appetite was normal. Physical examination showed a pulse rate of 102 beats per minute (bpm); blood pressure $(\mathrm{BP})=120 / 70 \mathrm{~mm} \mathrm{Hg}$; apex beat $(\mathrm{AB})=5$ th left intercostal space, mid-clavicular line (LICS MCL); and heart sounds (HS) = normal S1 and S2. There was bilateral exophthalmos. There was no dwarfing, no brachydactyly, no abnormal facies, and no other features suggestive of pseudohypoparathyroidism or DiGeorge syndrome. Cardiac and other systemic examinations were normal.

Anterior neck ultrasound showed a bilateral mildly enlarged diffuse thyroid gland. Corrected serum calcium level $\left(\mathrm{Ca}^{++}=1.95\right.$ $[2.2-2.7] \mathrm{mmol} / \mathrm{L})$, serum phosphate $\left(\mathrm{PO}_{4}=0.8[0.6-1.3] \mathrm{mmol} / \mathrm{L}\right)$, alkaline phosphatase, 24-h urinary calcium, magnesium, other electrolytes, and urea were within normal limits. Complete blood count was normal, and erythrocyte sedimentation rate (ESR) was $44 \mathrm{~mm} / \mathrm{h}$. Subsequently, thyroid function test (TFT) was done, which showed the following results: tri-iodothyronine $(\mathrm{T} 3)=12.5$ $(0.61-1.57) \mathrm{ng} / \mathrm{mL}$, levothyroxine $(\mathrm{T} 4)=17.7(2.17-3.34) \mathrm{ng} / \mathrm{mL}$, and thyroid-stimulating hormone $(\mathrm{TSH})=0.03(0.4-6.0) \mu \mathrm{IU} / \mathrm{mL}$. TSH receptor antibody test was not done due to financial difficulties; however, clinical diagnosis of Graves' disease was established by the presence of bilateral exophthalmos and diffusely enlarged thyroid gland [12]. X-ray of the long bones and pelvis showed no evidence of osteomalacia.

The patient was subsequently treated with carbimazole, then weaned off calcium supplements 3 months later. She had no episode of carpopedal spasm nor biochemical hypocalcemia 10 months after commencement of carbimazole.

\section{Case Report 2}

SM is a 42 -year-old woman who presented with a recurrent history of hypocalcemic tetany over the previous 20 months. She was treated with intravenous calcium during each presentation, then maintained on calcium and vitamin D supplements.

There was a history of palpitations, but no heat intolerance and no significant weight loss. Physical examination showed PR $=98$ bpm, $\mathrm{BP}=130 / 88 \mathrm{~mm} \mathrm{Hg}, \mathrm{AB}=5$ th LICS MCL, and HS $=$ normal S1 and S2. There was bilateral exophthalmos. There was no dwarfing, no brachydactyly, no abnormal facies, and no other features suggestive of pseudohypoparathyroidism or DiGeorge syndrome. Cardiac and other systemic examinations were not contributory.

Anterior neck ultrasound showed a bilateral mildly enlarged thyroid gland. Corrected serum calcium level $\left(\mathrm{Ca}^{++}=1.82[2.2-\right.$ $2.7] \mathrm{mmol} / \mathrm{L})$, serum phosphate $\left(\mathrm{PO}_{4}=1.4[0.6-1.3] \mathrm{mmol} / \mathrm{L}\right)$, alkaline phosphatase, 24-h urinary calcium, magnesium, other electrolytes, and urea were within normal limits. Complete blood count showed relative lymphocytosis, and ESR was $58 \mathrm{~mm} / \mathrm{h}$. TFT showed T3 $=19.7(0.61-1.57) \mathrm{ng} / \mathrm{mL}, \mathrm{T} 4=31.3(2.17-3.34) \mathrm{ng} /$ $\mathrm{mL}$, and TSH $=0.02(0.4-6.0) \mu \mathrm{IU} / \mathrm{mL}$. TSH receptor antibody test was not done due to financial difficulties; however, clinical diagnosis of Graves' disease was established by the presence of bilateral exophthalmos and diffusely enlarged thyroid gland [12]. X-ray of the long bones and pelvis showed no evidence of osteomalacia.

The patient was subsequently treated with carbimazole, then weaned off calcium and vitamin supplements 3 months later. She has remained normocalcemic 1 year after commencement of antithyroid medication.

\section{Case Report 3}

FG is a 34 -year-old para $3+0,3$ alive, who presented with severe carpopedal spasm at 2 weeks postpartum. There was recurrent history of carpopedal spasm during previous postpartum periods. She was treated with intravenous calcium and maintained on calcium and vitamin supplements. She was weaned off the supplements at about 6 months following each childbirth.

Examination revealed a bilateral mildly enlarged diffuse thyroid gland, with no exophthalmos. Other findings included PR $=108$ bpm, $\mathrm{BP}=134 / 84 \mathrm{~mm} \mathrm{Hg}, \mathrm{AB}=5$ th LICS MCL, and HS = normal S1 and S2. There was no dwarfing, no brachydactyly, no abnormal facies, and no other features suggestive of pseudohypoparathyroidism or DiGeorge syndrome. Other systems were normal.

Laboratory investigations showed a corrected serum calcium of $0.8(2.2-2.7) \mathrm{mmol} / \mathrm{L}, \mathrm{PO}_{4} 1.0(0.6-1.3) \mathrm{mmol} / \mathrm{L}$, alkaline phosphatase, 24-h urinary calcium, magnesium, other electrolytes and urea were within normal limits. Other laboratory results included a normal complete blood count, and ESR was $38 \mathrm{~mm} / \mathrm{h}$. TFT showed T3 $=12.3(0.61-1.57) \mathrm{ng} / \mathrm{mL}, \mathrm{T} 4=14.6(2.17-3.34) \mathrm{ng} /$ $\mathrm{mL}$, and TSH $=0.05(0.4-6.0) \mu \mathrm{IU} / \mathrm{mL}$. A diagnosis of recurrent severe hypocalcemia secondary to postpartum hyperthyroidism was made. After initial resuscitation, she was maintained on calcium and vitamin D supplements, then commenced on carbimazole. She was weaned off calcium/vitamin D supplements at 2 months, then carbimazole was stopped after 1 year of therapy. Clinical and biochemical normocalcemia and euthyroidism were sustained at 6-month follow-up.

\section{Discussion}

This is a case series of recurrent hypocalcemic tetany as the initial presentation in individuals with hyperthyroidism. Literature review showed that hypercalcemia 
has been reported much more than hypocalcemia in hyperthyroidism. Very few studies have reported hypocalcemia as a finding in hyperthyroidism [7-9]. Peculiar to our cases is the fact that recurrent hypocalcemic tetany was the first presentation of these women who were eventually diagnosed with hyperthyroidism. In addition, the recurrence of hypocalcemic tetany ceased after the treatment of hyperthyroidism, which is in keeping with findings of the few studies that reported same [5-7].

The effect of thyrotoxicosis on bone mineral metabolism of our cases may have aggravated total body calcium depletion due to underlying vitamin $\mathrm{D}$ deficiency or negative calcium balance, as was postulated by Yamashita et al. [8]. For instance, the increased physiologic calcium requirements for case 3 who went through pregnancy and was breastfeeding provided a background of body calcium depletion that was aggravated by postpartum thyrotoxicosis. Some authors have also reported that dark skin pigmentation is a risk factor for vitamin $\mathrm{D}$ deficiency due to inefficient conversion of skin 7-dehydrocholesterol to vitamin D [9]. All the 3 cases reported are dark-skinned women who may have subclinical vitamin $\mathrm{D}$ deficiency that was aggravated by the disordered calcium metabolism of thyrotoxicosis.

In a different vein, the findings of relative lymphocytosis and elevated ESR in case 2 may point to the presence of autoimmune thyroid and parathyroid diseases. Hyperphosphatemia, though mild, and hypocalcemia support the possibility of hypoparathyroidism in case 2 . This may have contributed to worsening of the disordered calcium metabolism of thyrotoxicosis. Furthermore, association of idiopathic hypoparathyroidism with hyperthyroidism can be supported by the presence of a normal serum magnesium level, absence of roentgenologic findings of osteomalacia, absence of renal insufficiency, absence of diarrhea, and absence of signs of pseudohypoparathyroidism like brachydactyly and dwarfing.

Idiopathic hypoparathyroidism (rare condition) may occur isolated or as a component of familial polyglandular endocrinopathy type 2 (mucocutaneous candidiasis, Addison's disease, or hypoparathyroidism). However, contrary to our cases, the syndrome usually present in childhood, and our cases did not have clinical features suggestive of Addison's disease or mucocutaneous candidiasis. Other rare causes of hypoparathyroidism that can be considered especially for case 2 (hypocalcemia and hyperphosphatemia) include Wilson's disease due to copper deposition in parathyroid gland, though slit-lamp examination did not show Kayser-Fleischer rings. DiGeorge syndrome is another rare cause of hypoparathyroidism, but none of our cases had cardiac defects, abnormal facies, or cleft palate.

Another possible mechanism contributing to hypocalcemia in especially case 2 (with mild hyperphosphatemia) includes compression of the blood vessels supplying the parathyroid glands by an enlarged thyroid gland, leading to ischemic necrosis of the parathyroid glands and thereby development of hypoparathyroidism. Furthermore, gastrointestinal malabsorption linked to thyrotoxicosis can aggravate hypocalcemia via steatorrhea (vitamin D deficiency) or direct calcium malabsorption. However, the vitamin D deficiency pathway was unlikely due to the normal serum phosphate and alkaline phosphatase levels.

From the various literature reviews and this case series, one may conclude that disordered calcium metabolism in thyrotoxicosis may manifest in successive phases of hypercalcemia-normocalcemia-hypocalcemia. The rate at which individual thyrotoxic patient navigates these phases may be directly proportional to the background vitamin $\mathrm{D}$ deficiency, negative calcium balance, and bone mineral density. Limitations of this study include the inability to measure serum $25(\mathrm{OH}) \mathrm{D}$, parathormone levels, and bone mineral density.

\section{Conclusion}

Recurrent hypocalcemia in selected persons should raise our index of suspicion to proactively pursue the diagnosis of hyperthyroidism. The pursuit of the diagnosis of thyrotoxicosis should be on selected patients with no obvious cause of recurrent hypocalcemia and with at least sustained tachycardia of no obvious cause, as may be seen in apathetic thyrotoxicosis. The recurrence of hypocalcemia ceased after the treatment of hyperthyroidism in all the cases reported.

Further research is needed to investigate our hypothesis that disordered calcium metabolism in thyrotoxicosis may manifest in successive phases of hypercalcemia-normocalcemia-hypocalcemia. The rate at which each thyrotoxic patient traverses these phases may be directly proportional to the background vitamin D deficiency, negative calcium balance, and bone mineral density.

\section{Acknowledgements}

Our gratitude to the staff of Medical Records Department of Federal Medical Center Azare for assisting us with the retrieval of the required case files and records.
Lawal/Dahuwa/Abdullahi/Anumah 


\section{Statement of Ethics}

Ethical approval was given by the Human Research and Ethics Committee of Federal Medical Center Azare. Written consent was obtained from the patients before writing this article for publication.

\section{Conflict of Interest Statement}

There was no conflict of interest in the writing of this article.

\section{Funding Sources}

Funding was strictly personal.

\section{Author Contributions}

Y.L. conceptualized the idea of this article, collected data, and wrote this article. U.F.D. was involved in the conceptualization, writing, and editing. I.A. and F.E.A. were involved in the conceptualization and editing of this article.

\section{Availability of Data and Material}

All data generated or analyzed during this study are included in this article. Further enquiries can be directed to the corresponding author.

\section{References}

1 Dimayuga DCM, Villa ML. SAT-336 an unusual case of symptomatic hypercalcemia from graves' disease in a young Filipino female. J Endocr Soc. 2020 May 8;4(Suppl 1): SAT-336.

2 Yan D, Xu Y, Li LX. The coexistence of hypercalcemia, osteoporosis and thymic enlargement in graves' disease: a case report. BMC Endocr Disord. 2020 Jun 30;20(1):97.

3 Liu J, Tang X, Cheng J, Yang X, Wang Y. Persistent arthralgia, vomiting and hypercalce$\mathrm{mia}$ as the initial manifestations of hyperthyroidism: a case report. Mol Clin Oncol. 2017 Feb;6(2):258-60.

4 Chen K, Xie Y, Zhao L, Mo Z. Hyperthyroidism-associated hypercalcemic crisis: a case report and review of the literature. Medicine. 2017 Jan;96(4):e6017.
5 Mat Ali MH, Tuan Ismail TS, Wan Azman WN, Yaacob NM, Yahaya N, Draman N, et al. Comparison of vitamin D levels, bone metabolic marker levels, and bone mineral density among patients with thyroid disease: a CrossSectional Study. Diagnostics. 2020 Dec 11; 10(12): 1075.

6 Dhananwal D, Dixit V, Marwaha V. Bone mineral homeostasis and bone mineral density in patients with hyperthyroidism after vitamin D therapy. India: UGC, Major Research Project; 2011. [Google Scholar]

7 Udayakumar N, Chandrasekaran M, Rasheed $\mathrm{MH}$, Suresh RV, Sivaprakash S. Evaluation of bone mineral density in thyrotoxicosis. Singapore Med J. 2006;47:947-50.

8 Yamashita H, Noguchi S, Takatsu K, Koike E, Murakami T, Watanabe S, et al. High preva- lence of vitamin D deficiency in Japanese female patients with Graves' disease. Endocr J. 2001;48:63-9.

9 Dhanwal DK. Thyroid disorders and bone mineral metabolism. Indian $\mathrm{J}$ Endocrinol Metab. 2011 Jul;15(Suppl 2):S107-12.

10 Macfarlane IA, Mawer EB, Berry J, Hann J. Vitamin D metabolism in hyperthyroidism. Clin Endocrinol. 1982;17:51-9.

11 Mosekilde L, Lund B, Sorensen OH, Christensen MS, Melsen F. Serum-25-hydroxycholecalciferol in hyperthyroidism. Lancet. 1977; 1:806-7.

12 Bell L, Hunter AL, Kyriacou A, Mukherjee A, Syed AA. Clinical diagnosis of Graves' or non-Graves' hyperthyroidism compared to TSH receptor antibody test. Endocr Connect. 2018 Mar 12;2017:7354673. [Medline]. 